$\overline{\text { Original }}$

\title{
Survival of Foodborne Pathogens in Grain Products and the Effect of Catechins
}

(Received November 7, 2008)

\author{
Junko Ui ${ }^{1}$, Kazuo Kondo ${ }^{1}$, Takuo Sawada ${ }^{2}$ and Yukiko Hara-Kudo ${ }^{3, *}$ \\ ${ }^{1}$ Institute of Environmental Science for Human Life, Ochanomizu University: \\ 2-1-1 Ohtsuka, Bunkyo-ku, Tokyo 112-8610, Japan; \\ 2 Nippon Veterinary and Life Science University: 1-7-1 Kyonancho, \\ Musashinoshi 180-0023, Japan; \\ ${ }^{3}$ Division of Microbiology, National Institute of Health Sciences: \\ 1-18-1 Kamiyoga, Setagaya-ku 158-8501, Tokyo, Japan; \\ * Corresponding author
}

\begin{abstract}
We investigated the survival of bacterial pathogens in grain products. Salmonella Enteritidis and Staphylococcus aureus survived for more than 3 weeks in grain flakes. Although S. Enteritidis in grain flakes reached a level of less than $10^{2} \mathrm{CFU} / \mathrm{g}$, the surviving organisms grew rapidly in milk and reached $10^{9} \mathrm{CFU} / \mathrm{g}$ after 25 -hr incubation. When catechins were present on the grain flakes, the numbers of $S$. Enteritidis and $S$. aureus were significantly decreased. In addition, catechins reduced the survival and growth of S. Enteritidis, S. aureus and Bacillus cereus when added to cooked grain products. This study indicates that S. Enteritidis, S. aureus and Bacillus can survive for a long time in grain products and that catechins impair their survival and growth.
\end{abstract}

Key words: foodborne pathogens; grain products; catechins

\section{Introduction}

Grain powders, such as corn flour and soy powder, may be contaminated with Salmonella ${ }^{1)}$ from animals $^{2), 3)}$ and soil bacilli4). However, bacteria can not grow in grain products, such as grain flakes and cooked grain, because the manufacturing process includes heating and drying ${ }^{5}$. Nevertheless, the spores of Bacillus cereus may survive after pasteurization during food processing. Therefore, during the storage of cooked foods under optimal growth conditions, the spores may germinate and then the vegetative cells grow to cause foodborne infections ${ }^{6}$. Grain products associated with foodborne infections have also been reported; examples include grain flakes contaminated with Salmonella ${ }^{7), 8)}$, dumpling contaminated with Staphylococcus aureus ${ }^{9)}$ and adzuki bean paste contaminated with $B$. cereus ${ }^{10)}$. In Japan, 141 cases of foodborne infections (123 cases of Salmonella Enteritidis and 18 cases of S. aureus) associated with adzuki bean paste, rice cake or dumpling have occurred ${ }^{11)}$. To find a way to control microorganisms in food, we evaluated catechins commonly found in tea extracts in this study. Catechins have antibacterial activity against pathogens such as pathogenic Vibrio species ${ }^{12)-14)}$, S. aureus ${ }^{15)}$, Escherichia coli $\mathrm{O} 157: \mathrm{H}^{16)}$, S. Enteritidis ${ }^{17)}$, and vegetative cells and spores of Clostridium botulinum ${ }^{18)}$. The antibacterial activity is attributed to the galloylester and pyrogallol moieties of the catechins, and the activity attributed to catechol and pyrogallol appears to be equal to that of the galloylester ${ }^{19)}$. Despite many reports on the antibacterial activity of catechins, there has been no report on the use of catechins as antibacterial food additives.

In the present study, we investigated the survival of bacterial pathogens in grain flakes. In addition, the antibacterial activities of catechins against the bacterial pathogens in the grain flakes, and the inhibition of bacterial growth were examined during storage of cooked grain products and grain flakes.

\section{Materials and Methods}

\section{Preparation of food samples.}

Three kinds of grain flakes: corn flakes, brown rice flakes and wheat bran flakes were purchased in retail shops in Tokyo. We dipped the corn flakes in solutions of catechins from green tea (Sanfenone, Taiyo Chemical Co., Ltd., Yokkaichi, Japan) at concentration of 1.0, 5.0 and $50 \mathrm{mg} / \mathrm{mL}$ and catechins were bound to the surface of the flakes at the final concentrations of 0, 0.1, 0.5 and $5.0 \mathrm{mg} / \mathrm{g}$, respectively. We found that $1 \mathrm{~g}$ of grain flakes absorbed $0.1 \mathrm{~mL}$ of catechin solution. The food treated with catechins was stored at $25^{\circ} \mathrm{C}$ until used for experiments. The following three kinds of cooked grain products prepared in the laboratory were treated with catechins at the concentration of $1 \mathrm{mg} / \mathrm{g}$ or not treated. A portion $(1 \mathrm{~mL})$ of catechin solution at the concentration of $100 \mathrm{mg} / \mathrm{mL}$ was added to $100 \mathrm{~g}$ of cooked food 
sample, i.e., adzuki bean paste (adzuki bean powder 180 $\mathrm{g}$, sugar $300 \mathrm{~g}$ and water $500 \mathrm{~mL}$ ), shiratama dumpling (glutinous rice powder $50 \mathrm{~g}$, rice powder $50 \mathrm{~g}$ and water $100 \mathrm{~mL}$ ) and warabimochi: a jelly-like confection made from starch (sweet potato starch $85 \mathrm{~g}$, kudzu vine starch $10 \mathrm{~g}$, bracken starch $5 \mathrm{~g}$, sugar $50 \mathrm{~g}$ and water $500 \mathrm{~mL})$.

\section{Strains and culture}

S. Enteritidis (from liquid egg: strain no. SEC 315), S. aureus (American Type Culture Collection: ATCC 25923) and Bacillus cereus (Japan Culture Microorganisms: JCM2152) were used. S. Enteritidis, S. aureus and $B$. cereus were cultured in $10 \mathrm{~mL}$ of tryptic soy broth (TSB; Becton Dickinson, Sparks, MD) for $24 \mathrm{hr}$ at $37^{\circ} \mathrm{C}$. The cultures were centrifuged at $3,000 \times g$ for $15 \mathrm{~min}$ at $4^{\circ} \mathrm{C}$. The cell pellet of each strain was suspended in phosphate-buffered saline (PBS; Nissui Co., Tokyo, Japan). The procedure was repeated twice to wash the cells. Finally the cells of each strain were suspended in $10 \mathrm{~mL}$ of PBS. These bacterial suspensions were used for inoculation of grain flakes. A $10^{4}$-fold dilution was used as the bacterial solution for inoculation of cooked grain products. To confirm the inoculation level of the bacterial strains, the cultures were diluted with PBS to $10^{-6}$. A $0.1 \mathrm{~mL}$ aliquot was plated onto tryptic soy agar (TSA; Becton Dickinson). After incubation at $37^{\circ} \mathrm{C}$ for $24 \mathrm{hr}$, the number of colonies was counted.

\section{Microbiological quality of the food sample}

Aerobic bacteria in the food samples were counted and it was confirmed that the samples were not contaminated with Salmonella, S. aureus and B. cereus as follows. Samples (10 g) were homogenized with $90 \mathrm{~mL}$ of PBS with a stomacher (Seward Co., Ltd., London, UK) for 1 min or a pulsifier (Microgen Bioproducts Ltd., UK) as a vibratory mixer for $15 \mathrm{sec}$. To count aerobic bacteria, $0.1 \mathrm{~mL}$ of the homogenate and each of serial 10 -fold dilutions in PBS were plated onto nutrient agar (NA; Nissui) and incubated at $37^{\circ} \mathrm{C}$ for $24 \mathrm{hr}$. The food samples were confirmed not to be contaminated with Salmonella, S. aureus and B. cereus using homogenate in BPW for $S$. Enteritidis, TSB with $7.5 \% \mathrm{NaCl}$ for $S$. aureus and TSB with polymixin (50 unit/mL) for $B$. cereus. After incubation at $37^{\circ} \mathrm{C}$ for $18 \mathrm{hr}$, the enrichment cultures of $S$. Enteritidis, $S$. aureus and B. cereus were streaked onto CHROMagar Salmonella (CHROMagar, Paris, France), Mannitol salt agar (Eiken Chemical, Tokyo, Japan) and NGKG (Niussi), respectively. Colonies were observed after incubation at $37^{\circ} \mathrm{C}$ for $24 \mathrm{hr}$.

\section{Survival of S. Enteritidis and S. aureus in grain flakes}

Corn flakes, brown rice flakes and wheat bran flakes were transferred to a stomacher bag in $10 \mathrm{~g}$ amounts and inoculated with $S$. Enteritidis or $S$. aureus solution $(0.1 \mathrm{~mL})$ at the inoculation level of approximately $10^{6}$ $\mathrm{CFU} / \mathrm{g}$. These samples were sealed with a heat-sealer and incubated at room temperature for 56 days, then homogenized with $90 \mathrm{~mL}$ of PBS in a pulsifier for $15 \mathrm{sec}$.
To determine the survival of pathogens, aliquots of 0.1 $\mathrm{mL}$ of serial 10 -fold dilutions was plated onto TSA as a non-selective agar medium and incubated at $37^{\circ} \mathrm{C}$ for 24 hr. Then the TSA-plated colonies of $S$. Enteritidis were transferred to CHROMagar Salmonella and the colonies of $S$. aureus were transferred to mannitol salt agar, from which they were picked up with needles for identification.

Growth of $S$. Enteritidis and S. aureus in grain flakes with milk. Samples (each $10 \mathrm{~g}$ ) of corn flakes, brown rice flakes and wheat bran flakes, inoculated with $S$. Enteritidis and $S$. aureus as described above, were kept at room temperature for 35 days to reach an undetectoble level by plating method described above. Then $90 \mathrm{~mL}$ of pasteurized milk purchased in a retail shop in Tokyo was poured onto the samples, and incubated at $25^{\circ} \mathrm{C}$ (room temperature) for $24 \mathrm{hr}$. To determine the growth of $S$. Enteritidis and $S$. aureus in grain flakes with milk, the cultures with milk were diluted $10^{-1}$ to $10^{-6}$-fold in PBS. The dilutions $(0.1 \mathrm{~mL})$ were plated onto CHROMagar Salmonella and mannitol salt agar medium (Oxoid) with 30\% egg yolk (EYMSA). Colonies were observed after incubation at $37^{\circ} \mathrm{C}$ for 24 $\mathrm{hr}$. The experiments were performed in quadruplicate.

Growth of S. Enteritidis, S. aureus and B. cereus in cooked grain products and the inhibitory effect of catechin. The cooked grain products (adzuki bean paste, shiratama dumpling and warabimochi) treated with/ without catechins were transferred to a stomacher bag (10 g) and inoculated with S. Enteritidis, S. aureus and $B$. cereus at the level of $10^{2} \mathrm{CFU} / \mathrm{g}$, and then incubated at 4 or $25^{\circ} \mathrm{C}$ for 3 days. To determine the growth of the pathogens in the cooked grain products and the inhibitory effect of catechins, samples were homogenized with $90 \mathrm{~mL}$ of PBS in a pulsifier for $15 \mathrm{sec}$ or a stomacher for $1 \mathrm{~min}$. Each serial 10 -fold dilution $(0.1 \mathrm{~mL})$ was plated onto XLD, EYMSA and NGKG. Colonies were observed after incubation at $37^{\circ} \mathrm{C}$ for $24 \mathrm{hr}$. The experiments were performed in quadruplicate.

\section{Enrichment of food homogenate and plating of enrichment culture}

To confirm that the population of each pathogen was below the detection limit, the homogenates $(100 \mathrm{~mL})$ of grain flakes and cooked grain food were added to 100 $\mathrm{mL}$ of enrichment broth prepared at double strength: buffered peptone water (BPW; Oxoid, Basingstoke, Hampshire, UK) for S. Enteritidis; TSB with $7.5 \% \mathrm{NaCl}$ for $S$. aureus; TSB with polymixin (50 unit $/ \mathrm{mL}$ ) for $B$. cereus. Incubation was carried out at $37^{\circ} \mathrm{C}$ for $18 \mathrm{hr}$. The enrichment cultures for $S$. Enteritidis, S. aureus and $B$. cereus were streaked onto XLD (Oxoid), EYMSA and NGKG, respectively. Colonies were observed after incubation at $37^{\circ} \mathrm{C}$ for $24 \mathrm{hr}$.

Confirmation of $S$. Enteritidis, S. aureus and B. cereus. A portion of the colonies suspected to be Salmonella on CHROMagar Salmonella and XLD was tested for agglutination with a Salmonella antibody kit (the Salmonella latex test, Unipath, Oxoid). A portion of the suspected $S$. 
aureus colonies was tested for coagulase and confirmed to be Gram-positive cocci with the Gram stain and microscopy. A portion of the suspected B. cereus colonies was tested for catalase, VP reaction, growth under anaerobic conditions and growth at $\mathrm{pH}$ 5.7. The enterotoxin of $B$. cereus in culture was detected using a reverse passive latex agglutination kit (CRET-RPLA, Denka Seiken, Tokyo, Japan). The experiments were performed in quadruplicate.

\section{Statistical analysis.}

Significant differences between bacterial populations incubated with catechins and without catechins were analyzed by using Student's $t$-test. A $p$ value $<0.05$ was taken to be significant.

\section{Results and Discussion}

Aerobic bacteria counts in corn flakes, adzuki bean paste, siratama dumpling and warabimochi were less than $1.9 \mathrm{log}$, cfu/g though those in brown rice flakes
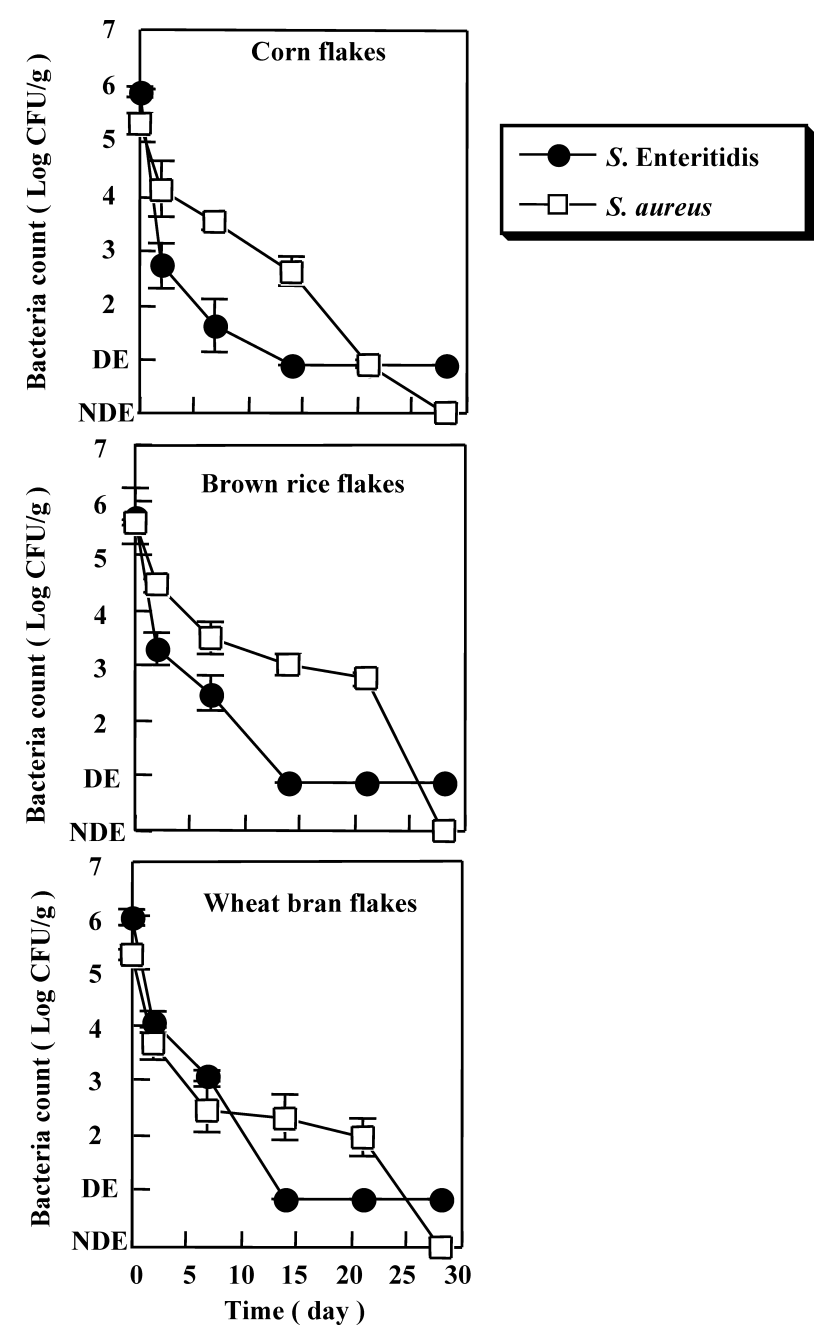

Fig. 1. Survival of $S$. Enteritidis and $S$. aureus in grain flakes.

Bars represent standard errors. DE: detected in enrichment culture but not detected by the plating method, NDE: not detected in enrichment culture. and wheat bran flakes were 3.9 and $5.2 \mathrm{log}$, cfu/g, respectively. Food samples used in this study were confirmed not to be contaminated with Salmonella, S. aureus or B. cereus.

In the three kinds of grain flakes, the population of $S$. Enteritidis quickly decreased and the organism was undetectable by a plating method at day 14, but remained detectable by enrichment at day 28 (Fig. 1). The population of $S$. aureus decreased and became undetectable by enrichment at day 28. In corn flakes and brown rice flakes, the decrease of the $S$. aureus population was slower than that of the $S$. Enteritidis population. It was demonstrated that foodborne pathogens can survive in grain flakes.

Grain flakes are usually consumed very soon after milk is poured on them. But children and old people may allow the grain flakes to soften in milk, and may leave them for a long time before eating them. In this study, milk was poured into the three kinds of grain

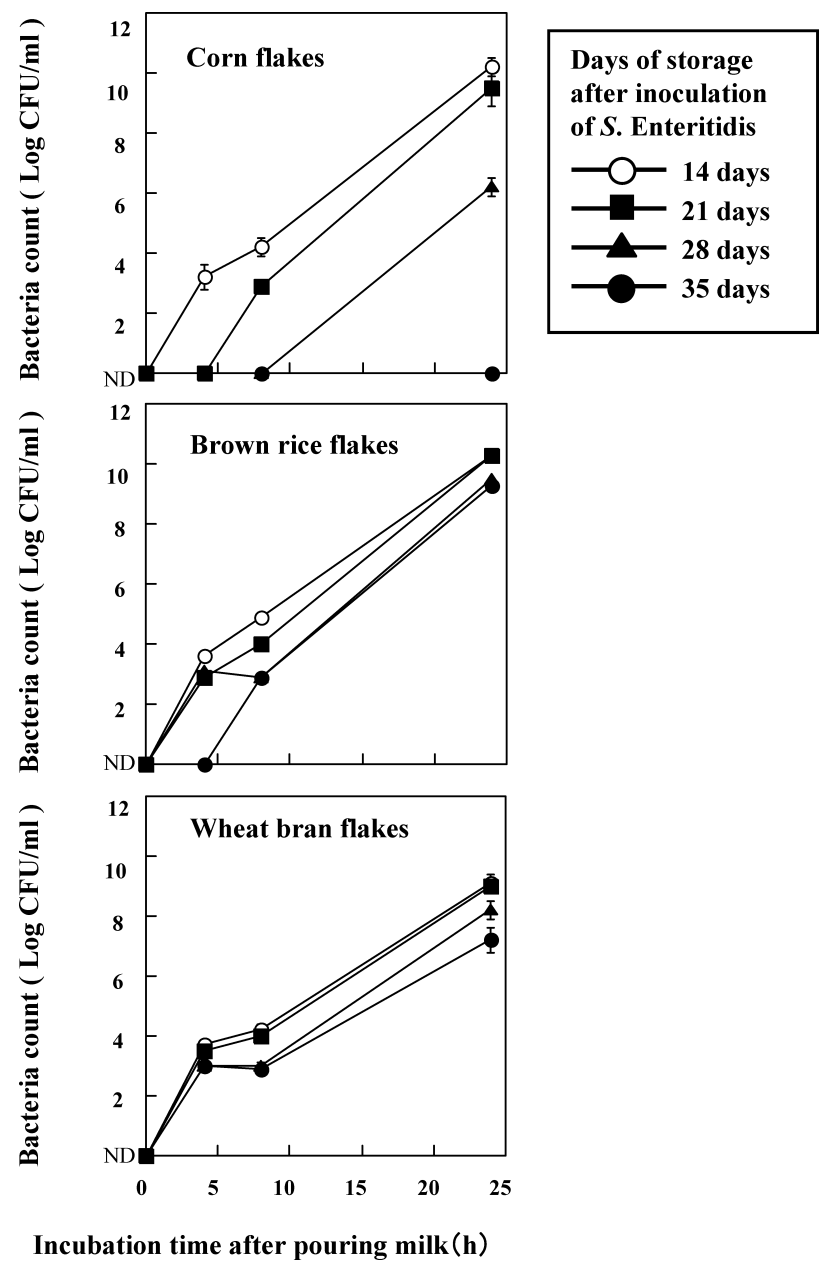

Fig. 2. Growth of $S$. Enteritidis in grain flakes with milk.

Milk was poured onto grain flakes in which the population of $S$. Enteritidis was undetectable after storage, and incubated at $25^{\circ} \mathrm{C}$. Bars represent standard errors. ND: not detected by plating method. : $\bigcirc, 14$ days; $\boldsymbol{\square}, 21$ days; $\boldsymbol{\Delta}, 28$ days and $\mathbf{0}, 35$ days. 
flakes stored for 14, 21, 28 and 35 days after inoculation with $S$. Enteritidis and S. aureus. S. Enteritidis grew in each kind of grain flakes with milk (Fig. 2), but S. aureus did not grow at all (data not shown). In corn flakes and brown rice flakes, the lag phase of $S$. Enteritidis was long (Fig. 2). In wheat bran flakes, $S$. Enteritidis quickly grew in all samples after 4-hr incubation (Fig. 2). The results indicate that small numbers of $S$. Enteritidis in grain flakes could grow in milk and may be a cause of food poisoning.

The number of $S$. Enteritidis was below the detection limit by enrichment at day 7 in corn flakes treated with catechins at the concentrations of 0.5 and $5.0 \mathrm{mg} / \mathrm{g}(p<$ 0.01 ) (Fig. 3). The population of $S$. aureus was undetectable by enrichment at day 7 at the concentration of more than $0.1 \mathrm{mg} / \mathrm{g}(p<0.01)$ (Fig. 3). Catechins are known to have antibacterial activity ${ }^{20}$. In this study, catechins impaired the growth of foodborne pathogens in grain flakes and cooked grain products. Catechin treatment might be an effective way to interfere with the survival of foodborne pathogens in corn flakes and other dried grain products.

\section{(a) $S$. Enteritidis}
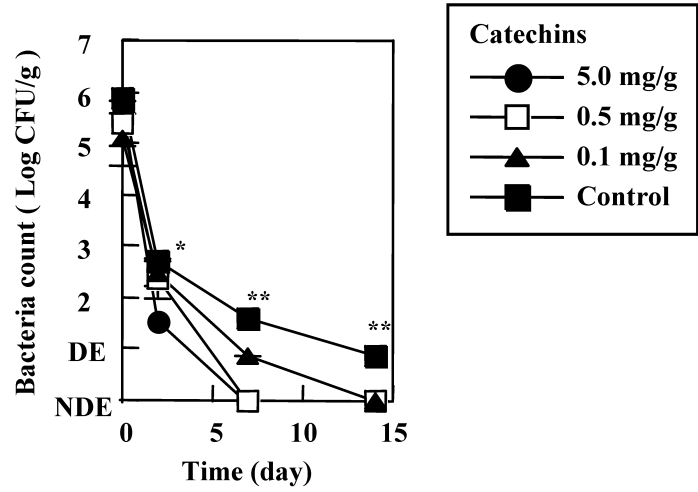

(b) S. aureus

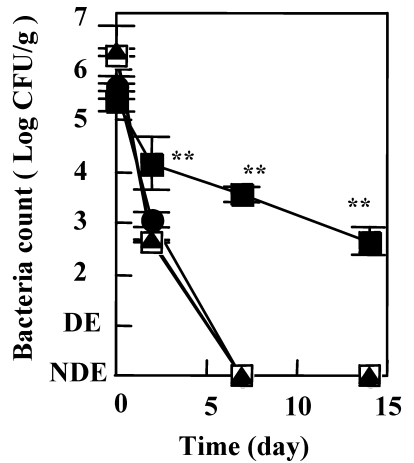

Fig. 3. Inhibitory effect on the growth of $S$. Enteritidis and $S$. aureus in corn flakes treated with catechin.

Bars represent standard errors. ${ }^{*}$ Control vs. 0.1 and $0.5 \mathrm{mg} / \mathrm{g}: p<0.05$, control vs. $5.0 \mathrm{mg} / \mathrm{g}: p<$ 0.01; ${ }^{* *}$ Control vs. $0.1,0.5$ and $5 \mathrm{mg} / \mathrm{g}: p<0.01$. DE: detected in enrichment culture but not detected by the plating method, NDE: not detected in enrichment culture.
Adzuki bean paste, shiratama dumpling and warabimochi treated with catechins at the concentration of $1 \mathrm{mg} / \mathrm{g}$ were inoculated with $S$. Enteritidis, $S$. aureus and $B$. cereus, and incubated at 4 and $25^{\circ} \mathrm{C}$. In adzuki bean paste treated with catechins, S. aureus, $B$. cereus and $S$. Enteritidis were not detected by enrichment at 4 or $25^{\circ} \mathrm{C}$ (Fig. 4a). Although B. cereus incubated for 3 days at $4^{\circ} \mathrm{C}$ in shiratama dumpling grew in the enrichment broth, catechins significantly impaired the growth $(p<0.01)$ (data not shown). The populations of $S$. aureus and $B$. cereus in warabimochi treated with catechins were undetectable by enrichment after having been incubated at 4 and $25^{\circ} \mathrm{C}$ for a day $(p<0.01)$ (Fig. 4b). Catechins in warabimochi were more effective than in adzuki bean paste and shiratama dumplings, although the reason is not known. The protein content of warabimochi is less than those of adzuki bean paste and shiratama dumplings. Some components of adzuki bean paste and shiratama dumplings might inhibit the activity of the catechins.

The growth of S. aureus in warabimochi at $25^{\circ} \mathrm{C}$ was inhibited at day 3 (Fig. 4b) although S. aureus grew to ca. $5 \log \mathrm{CFU} / \mathrm{g}$ in adzuki bean paste. This might indicate that the high concentration of sugar in adzuki bean paste promoted the growth of $S$. aureus. On the other hand, the population of $B$. cereus in warabimochi increased to $c a$. $5 \log \mathrm{CFU} / \mathrm{g}$ after incubation for 1 day at
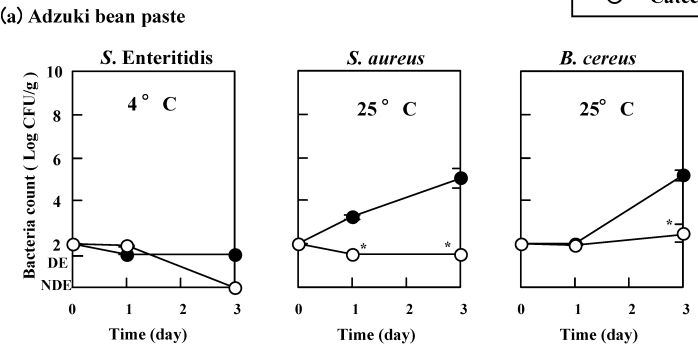

(b) Warabimochi

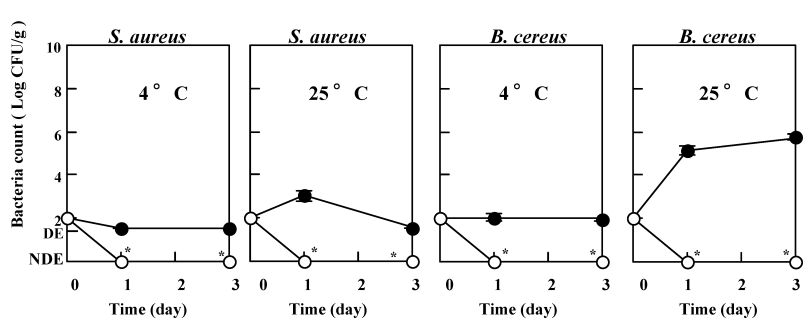

Fig. 4. Inhibitory effect of catechins on the growth of $S$. Enteritidis, S. aureus and B. cereus in cooked grain products.

(a) S. Enteritidis, S. aureus and B. cereus in adzuki bean paste, (b) B. cereus in shiratama dumpling after incubation for 3 days, (c) $S$. aureus and $B$. cereus in warabimochi. The concentration of catechins was $1 \mathrm{mg} / \mathrm{g}$. Temperature was 4 or $25^{\circ} \mathrm{C}$. Bars represent standard errors. ${ }^{*}$ Control $v s$. catechins $+: p<0.01$. DE: detected in enrichment culture but not detected by the plating method, NDE: not detected in enrichment culture. 
$25^{\circ} \mathrm{C}$, although that in adzuki bean paste did not change. Again, this might indicate that the high concentration of sugar in adzuki bean paste inhibited the growth of $B$. cereus.

In the present study, the antibacterial activities of catechins were examined in grain products during storage. The results suggest that catechins might be effective as a food additive. While there is no regulatory limit for catechins, the amount of catechins that can be added to food may be limited due to the change in the taste of the food. Because a synergistic effect between catechins and antibiotics was recently reported ${ }^{21)}$, a combination of catechins with other food additives might allow a decrease in the effective dose of food additives. The addition of catechins to food to control foodborne pathogens should be further investigated. In addition, grain flakes should be tested by enrichment, which is an effective procedure to detect small numbers of foodborne pathogens, because pathogens survive in grain flakes at a low contamination levels for a long time. Grain flakes also should be taken as soon as possible after pouring milk to avoid the possibility of growth of foodborne pathogens.

\section{References}

1) Richter, K. S., Dorneau, E., Eskridge, K. M., Rao, C. S. Microbiological quality of flour. Cereal Foods World, 38, 367-369 (1993).

2) Berghofer, L., Hocking, A., Miskelly, D., Jansson, E. Microbiology of wheat and flour milling in Australia. Int. J. Food Microbiol., 85, 137-149 (2003).

3) Vancauwenberge, J., Bothast, R., Kwolek, W. Thermal inactivation of eight Salmonella serotypes on dry corn flour. Appl. Environ. Microbiol., 42, 688-691 (1981).

4) Stabb, E. V., Jacobson, L. M., Handelsman, J. Zwittermicin A-producing strains of Bacillus cereus from diverse soils. Appl. Environ. Microbiol., 60, 4404-4412 (1994).

5) International Commission on Microbiological Specifications for Foods (ICMSF). Cereals and cereal products. In Microorganisms in foods. 6. Microbial ecology of food commodities. Kluwer Academic/Plenum Publishers, New York, 2005, p. 392-439.

6) Centers for Disease Control and Prevention. Bacillus cereus food poisoning associated with fried rice at two child day care centers-Virginia, 1993. Morb. Mortal Wkly. Rep., 43, 177-178 (1994).

7) Centers for Disease Control and Prevention. Multistate outbreak of Salmonella serotype Agona infections linked to toasted oats grain flakes-United States, April-May, 1998. Morb. Mortal. Wkly. Rep., 47, 462-464 (1998).

8) Zhang, G., Ma, L., Patel, N., Swaminathan, B., Wedel, S.,
Doyle, M. P. Isolation of Salmonella Typhimurium from outbreak-associated cake mix. J. Food Prot., 70, 9971001 (2007).

9) Infectious Disease Surveillance Center. An outbreak of Staphylococcus food poisoning caused by "dango", dumplings made from rice flour coated with syrup or bean jam, and "daifuku", rice cakes stuffed with sweet bean jam, November 2000-Shizuoka City. Infect. Agents Surv. Rep., 22, 192-193 (2001).

10) Infectious Disease Surveillance Center. A large-scale outbreak of vomiting-type food poisoning due to Bacillus cereus caused by "an-iri-mochi", rice cakes stuffed with sweet bean jam, at a nursery school, December 2001-Kumamoto City. Infect. Agents Surv. Rep., 23, 93-94 (2002).

11) Ministry of Health and Welfare, Japan. The reports on food poisoning in Japan. Japan Food Hygiene Association, Tokyo, Japan (1997). (in Japanese).

12) Hara, Y., Ishigami, T. Antibacterial activities of tea polyphenols against foodborne pathogenic bacteria. Nippon Shokuhin Kogyo Gakkaishi, 36, 996-999 (1989). (in Japanese)

13) Toda, M., Okubo, S., Ohnishi, R., Shimamura, T. Antibacterial and bactericidal activities of Japanese green tea. Nippon Saikingaku Zasshi, 44, 669-672 (1989). (in Japanese)

14) Toda, M., Okubo, S., Ikigai, H., Suzuki, T., Suzuki, Y., Shimamura, T. The protective activity of tea against infection by Vibrio cholerae O1. J. Appl. Bacteriol., 70, 109-112 (1991).

15) Toda, M., Okubo, S., Hiyoshi, R., Shimamura, T. The bactericidal activity of tea and coffee. Lett. Appl. Microbiol., 8, 123-125 (1989).

16) Hara-Kudo, Y., Okubo, T., Tanaka, S., Chu, D., Juneja, L. R., Saito, N., Sugita-Konishi, Y. Bacterial action of green tea extract and damage to the membrane of Escherichia coli O157 : H7. Biocontrol Sci., 6, 58-61 (2001).

17) Toda, M., Okubo, S., Hiyoshi, R., Ohnishi, T., Shimamura, T. The bactericidal activity of tea and coffee. Lett. Appl. Microbiol., 44, 669-672 (1989).

18) Hara, Y., Watanabe, M. Antibacterial activity of polyphenols against Clostridium botulinum. Nippon Shokuhin Kogyo Gakkaishi, 36, 951-955 (1989). (in Japanese)

19) Toda, M., Okubo, S., Ikigai, H., Shimamura, T. Antibacterial and anti-hemolysin activities of tea catechins and their structural relatives. Nippon Saikingaku Zasshi, 45, 561-566 (1990). (in Japanese)

20) Friedman, M., Henika, P. R., Levin, C. E., Mandrell, R. E., Kozukue, N. Antimicrobial activities of tea catechins and theaflavins and tea extracts against Bacillus cereus. J. Food Prot., 69, 354-361 (2006).

21) Shimamura, T., Zhao, W. H., Hu, Z. Q. Mechanism of action and potential for use of tea catechin as an antiinfective agent. Anti-Infective Agents in Med. Chem., 6, 57-62 (2007). 